\title{
Delay of vortex ring pinchoff by an imposed bulk counterflow
}

\author{
John O. Dabiria) and Morteza Gharib \\ Option of Bioengineering and Graduate Aeronautical Laboratories, California Institute of Technology, \\ Pasadena, California 91125
}

(Received 23 October 2003; accepted 21 January 2004; published online 8 March 2004)

\begin{abstract}
The Kelvin-Benjamin variational principle has been previously used to predict that vortex ring pinchoff can be delayed if the energy of the leading vortex ring is decreased during formation and/or the energy delivered by the vortex generator is increased. We present experimental results in which the former and latter energy effects were simultaneously accomplished by imposing a bulk axisymmetric counterflow during vortex ring formation. Measurements indicate that the formation number is retarded sufficiently to allow increased ingestion of fluid circulation by the leading vortex ring. This serves as a first demonstration of artificial manipulation of vortex ring formation for potential propulsive benefits in starting jets. (C) 2004 American Institute of Physics.
\end{abstract}

[DOI: $10.1063 / 1.1669353$ ]

Krueger ${ }^{1,2}$ recently measured the time-integrated force associated with vortex ring formation in a starting jet, and determined that its magnitude is greater than an equivalent steady jet. This result encourages one to imagine the possibility of exploiting arbitrarily large vortex rings for unsteady fluid transport or thrust generation.

The question of whether rings of arbitrary size can be created in practice was not examined until relatively recently. Gharib et al. ${ }^{3}$ studied the formation of vortex rings using a piston-in-cylinder arrangement. They demonstrated that at sufficiently large stroke length-to-diameter ratios, the size of the leading vortex ring can no longer be increased. Fluid ejected from the vortex generator subsequent to this limit forms an unstable trailing jet posterior to the ring. Krueger ${ }^{1}$ compared the time-integrated force due to vortex rings formed below and above the limiting stroke ratio, and showed that the force production is maximized as the limit is reached.

The discovery and characterization of a limiting process during vortex ring formation has generated substantial interest in the concept of optimal unsteady jet propulsion and methods to delay vortex ring pinchoff. Gharib et al. ${ }^{3}$ and others subsequently have characterized the limiting process as a manifestation of the Kelvin-Benjamin variational principle. ${ }^{4-7}$ In effect, the principle predicts the separation of the leading vortex ring from the trailing fluid supply (known as vortex ring pinchoff) when the vortex generator is no longer able to supply energy at a level sufficient for ring growth. Further, the principle dictates that the energy of an axis-touching ring, i.e., one with the core vorticity reaching the symmetry axis asymptotically, is maximized relative to other arrangements of the vorticity with the same impulse.

These developments suggest that in order to delay the pinchoff process, the energy required by the leading vortex ring must be decreased and/or the energy delivered by the vortex generator must be increased during the formation pro-

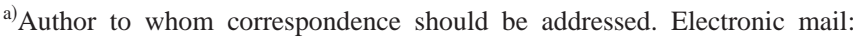
jodabiri@ caltech.edu
}

cess. The objective of these experiments is to demonstrate both strategies simultaneously, in order to suggest the possibility of achieving propulsive benefits from augmented leading vortex rings in starting jets.

By establishing bulk counterflow in the fluid surrounding a vortex generator, the energy of the forming vortex ring can be altered. Formally, the ring energy is given by ${ }^{8}$

$$
E_{\text {ring }}=\int_{V} \frac{1}{2} \mathbf{u}^{2} d V=2 \mathbf{U} \cdot \mathbf{I}+\int_{V}(\mathbf{u}-\mathbf{U}) \cdot \mathbf{r} \times \boldsymbol{\omega} d V,
$$

where $\mathbf{U}$ is the vortex velocity, I is the fluid impulse, $\mathbf{u}$ and $\boldsymbol{\omega}$ are the fluid velocity and vorticity, respectively, and the integration is taken over the ring volume. The first term in the last expression accounts for translational energy while the second term includes the energy of fluid motion in the vortex ring relative to its translating frame of reference. A bulk counterflow at speed $U_{c n}$ decreases the vortex ring translational velocity, resulting in a concomitant decrease in ring energy:

$$
E_{\text {ring }}=2\left(\mathbf{U}-U_{c n}\right) \cdot \mathbf{I}+\int_{V}(\mathbf{u}-\mathbf{U}) \cdot \mathbf{r} \times \boldsymbol{\omega} d V .
$$

The energy delivered by the vortex generator is also affected by counterflow, since the exit velocity in the energy equation is measured relative to the bulk velocity of the ambient fluid. Using a format similar to that of Shusser and Gharib, ${ }^{9}$

$$
E_{\text {generator }}=\frac{1}{8} \pi D^{2} L\left(U_{e}+U_{c n}\right)^{2},
$$

where $D$ and $L$ are the nozzle exit diameter and stroke length, respectively, and $U_{e}$ is the exit velocity of the fluid efflux from the vortex generator in a laboratory frame. Equations (2) and (3) suggest that counterflow both decreases the ring energy and increases the vortex generator energy, delaying ring pinchoff.

These hypotheses were tested using the apparatus of Fig. $1 .^{10}$ Operation is similar to that described by Krueger et al. ${ }^{11}$ for a coflowing configuration. Measurement of vortex ring 


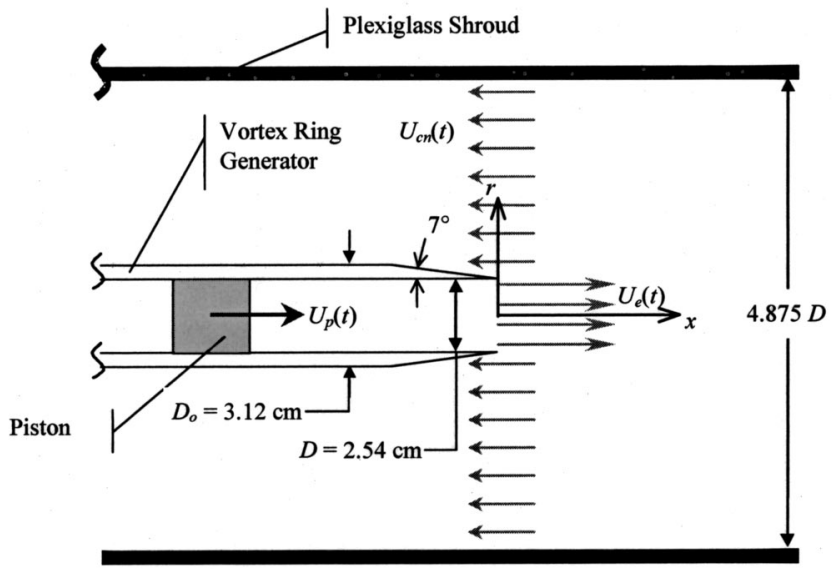

FIG. 1. Schematic of basic apparatus for counter-flow experiments (Ref. 10).

circulation was accomplished using digital particle image velocimetry (DPIV). ${ }^{12}$ Neutrally buoyant $20-\mu \mathrm{m}$ particles were seeded in the flow to scatter incident light from a 532-nm pulsed Nd:YAG laser. The flow field -8 diameters downstream of the nozzle exit and 2.4 diameters radially from the symmetry axis-was captured by a $1024 \times 1024$ pixel blackand-white charge-coupled device digital camera (Uniq Vision, Inc.) at $30 \mathrm{~Hz}$. This pixel resolution corresponds to a physical test section resolution of approximately 0.19 $\times 0.19 \mathrm{~mm}$, and was sufficient to resolve the vortex ring core vorticity distribution.

Vortex rings were generated for stroke length-todiameter ratios $L / D$ of 4 and 12, nominally, at a Reynolds number of 1400 based on the nominal piston speed (5.5 $\mathrm{cm} / \mathrm{s}$ ) and nozzle exit diameter $(2.54 \mathrm{~cm})$. The following counterflow protocols and corresponding test names were introduced: $\mathrm{CF} 0=$ no counterflow; $\mathrm{CF} 05-n=$ counterflow at one half of the nominal piston speed and a $n L / D$ delay in its initiation relative to the start of vortex ring formation (e.g., CF05-2 corresponds to a $2 L / D$ delay in counterflow); CFE = counterflow matched to the measured instantaneous $\mathrm{CF} 0$ vortex ring speed.

The counterflow changed the dynamics of vorticity flux without significantly altering the total circulation delivered by the vortex generator. This is demonstrated by the plots of circulation versus nondimensional time ${ }^{13} \hat{t}=\left(\bar{U}_{p}-\bar{U}_{c n}\right) t / D$ at stroke ratio 4, shown in Fig. 2.

For the stroke ratio 4, vortex ring pinchoff consistently occurred at nondimensional time near 3.6, where pinchoff was observed at all. This formation number lies in the range observed by Gharib et al. ${ }^{3}$ in the absence of bulk flow. Complete suppression of vortex ring pinchoff was accomplished for cases CF05-2 and CFE. The angled line in the figure separates trials in which pinchoff was observed (right hand side of line) and those in which all of the circulation ejected from the generator was ingested by the leading vortex ring. From this plot it appears that an increased rate of vorticity flux in nondimensional time is consistently correlated with sustained growth of the leading vortex ring.

It is clear that the delayed pinchoff in the CF05-2 and

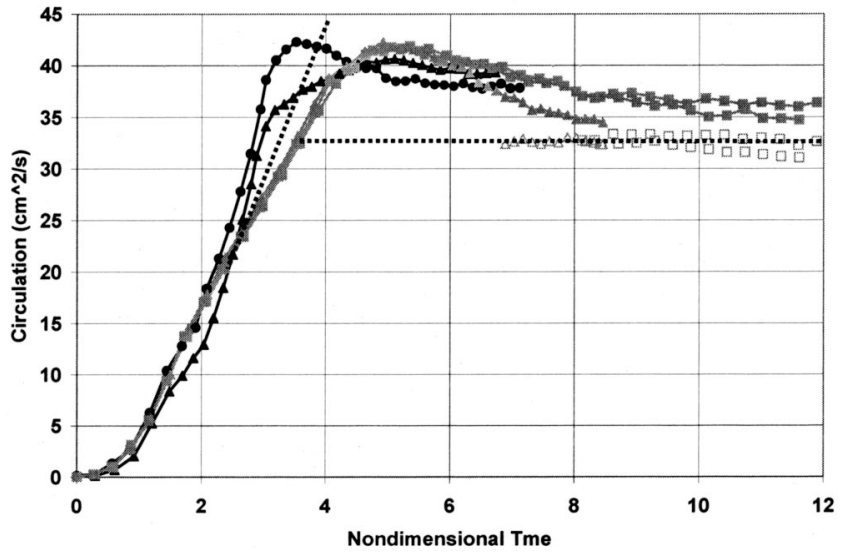

FIG. 2. Circulation versus nondimensional time for nominal stroke ratio of 4. Gray squares, CF0; black triangles, CF05-2; gray triangles, CF05-6; black circles, CFE. Open symbols represent circulation in pinchedoff leading vortex ring.

CFE trials is due in part to the counterflow being implemented prior to normal vortex ring pinchoff (i.e., $\hat{t}<3.6$ ). To be sure, the counterflow for CF05-6 is identical in magnitude to the CF05-2 case, but is initiated after pinchoff has already occurred, and therefore cannot affect the pinchoff dynamics. This is effectively demonstrated in Fig. 3(a), which plots the
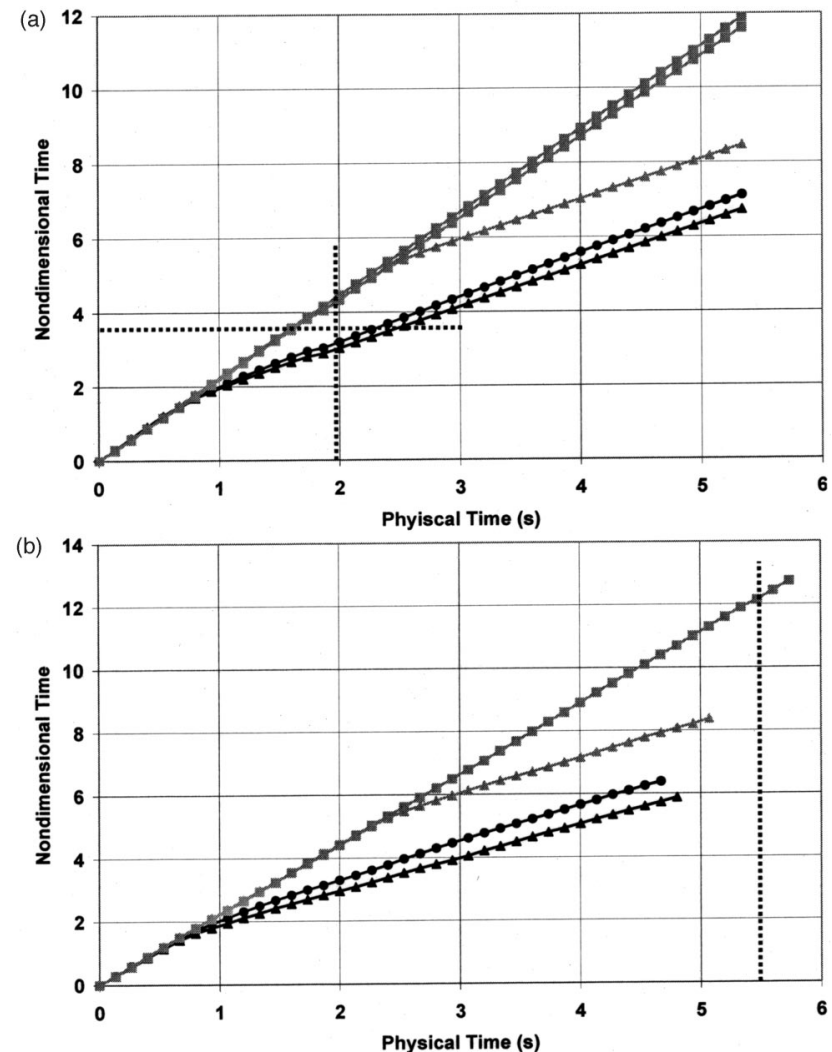

FIG. 3. Nondimensional time versus physical time. (a) Stroke ratio=4; (b) stroke ratio $=12$. Gray squares, $\mathrm{CF} 0$; black triangles, $\mathrm{CF} 05-2$; gray triangles, CF05-6; black circles, CFE. Vertical line denotes end of fluid ejection. Horizontal line in (a) denotes measured formation number for vortex ring pinchoff. 
nondimensional time versus real time and indicates the end point of fluid ejection by the vortex generator.

The trials observed to experience pinchoff of the leading vortex ring (i.e., $\mathrm{CF} 0$ and $\mathrm{CF} 05-6)$ reach the formation number (horizontal line) before the end point of fluid ejection. Accordingly, the leading vortex pinches off and a trailing jet is formed. The two cases in which counterflow was initiated prior to the normal pinchoff do not reach the formation number until after fluid ejection has terminated. Thus, the leading vortex ring is able to accommodate all of circulation from the vortex generator. Initiation of counter-flow is indicated by the abrupt decrease in curve slope for cases CF05-2, CF05-6, and CFE.

Similar efforts were made to delay vortex ring pinchoff at a stroke ratio of 12 . However, the counterflow protocols were unable to suppress the nondimensional time below the formation number for the entire duration of fluid ejection [Fig. 3(b)]. Hence, pinchoff of the leading vortex ring was observed in each trial. ${ }^{14}$

These results elucidate important principles governing vortex ring pinchoff in starting jets. Specifically, we have empirically demonstrated the sufficient condition for complete avoidance of vortex ring pinchoff that is suggested by the Kelvin-Benjamin variational principle: the formation time must be suppressed below the formation number until the end of fluid ejection. The use of a counterflow to reduce vortex ring energy and increase vortex generator energy is a simple but effective mechanism to exploit the salient limiting processes and achieve enhanced vortex ring formation. In the present case, the circulation of the vortex rings that were effectively manipulated by counterflow was increased by over $25 \%$ at peak circulation levels. The increased circulation persists at least $10 \%$ above normal levels even at later times (e.g., beyond nondimensional time 8), when a reduction from peak circulation levels occurs due to cancellation of vorticity at the symmetry axis and loss of vorticity into a small wake during the process of fluid entrainment. ${ }^{15}$ These dissipative mechanisms have been duly noted in both vortex ring experiments and numerical simulations. ${ }^{3,5}$

The counterflow technique described here is the first empirical demonstration of effective pinchoff delay. The authors intend to shift focus to methods of pinchoff delay that do not rely on external devices, such as might be accomplished by manipulation of parameters of the vortex generator itself. Because the Kelvin-Benjamin variational principle does not lend itself to immediate interpretation in terms of a physical vortex generator, we will rely on analyses similar to those introduced in Eq. (1)-(3) herein.

\section{ACKNOWLEDGMENT}

This research is supported under National Science Foundation Grant No. 0309671.

${ }^{1}$ P. S. Krueger, "The significance of vortex ring formation and nozzle exit overpressure to pulsatile jet propulsion," Ph.D. thesis, California Institute of Technology, Pasadena, CA, 2001.

${ }^{2}$ P. S. Krueger and M. Gharib, "The significance of vortex ring formation to the impulse and thrust of a starting jet," Phys. Fluids 15, 1271 (2003).

${ }^{3}$ M. Gharib, E. Rambod, and K. Shariff, "A universal time scale for vortex ring formation," J. Fluid Mech. 360, 121 (1998).

${ }^{4}$ P. G. Saffman, Vortex Dynamics (Cambridge University Press, Cambridge, 1992).

${ }^{5}$ K. Mohseni, H. Ran, and T. Colonius, "Numerical experiments on vortex ring formation," J. Fluid Mech. 430, 267 (2001).

${ }^{6} \mathrm{~K}$. Mohseni and M. Gharib, “A model for universal time scale of vortex ring formation,” Phys. Fluids 10, 2436 (1998).

${ }^{7} \mathrm{~K}$. Mohseni, "Statistical equilibrium theory for axisymmetric flows: Kelvin's variational principle and an explanation for the vortex ring pinchoff process," Phys. Fluids 13, 1924 (2001).

${ }^{8}$ P. G. Saffman, "The velocity of viscous vortex rings," Stud. Appl. Math. 49, 371 (1970).

${ }^{9}$ M. Shusser and M. Gharib, "Energy and velocity of a forming vortex ring," Phys. Fluids 12, 618 (2000).

${ }^{10}$ Figure 1 has been adapted from Ref. 11. The shroud has been modified to provide a longer viewing window in the axial direction, relative to the device in that reference.

${ }^{11}$ P. S. Krueger, J. O. Dabiri, and M. Gharib, "Vortex ring pinchoff in the presence of simultaneously initiated uniform background co-flow," Phys. Fluids 15, L49 (2003).

${ }^{12}$ C. E. Willert and M. Gharib, "Digital particle image velocimetry," Exp. Fluids 10, 181 (1991).

${ }^{13}$ This definition of the nondimensional time is consistent with the argument presented by Ref. 11, in which it is shown that the nondimensional vorticity flux should equal 0.5 irrespective of bulk motion in the ambient fluid. The overbar indicates a running time average of the associated quantity.

${ }^{14}$ The occurrence of vortex ring pinchoff at stroke ratio 12 could be observed clearly. This is despite the inability to quantify the formation number definitively, due to limited viewing range downstream of the vortex generator.

${ }^{15}$ T. Maxworthy, "The structure and stability of vortex rings," J. Fluid Mech. 51, 15 (1972). 